\title{
Photonic Wire Bonding and 3D Nanoprinting in Photonic Integration - from Lab Demonstrations to Production
}

\author{
C. $\operatorname{Koos}^{\left(1,2,3,{ }^{*}\right)}$, W. Freude $(1)$, S. Randel ${ }^{(1)}$, M. R. Billah ${ }^{(1,2)}$, M. Blaicher ${ }^{(1,2)}$, \\ P.-I. Dietrich ${ }^{(1,2,3)}$, T. Hoose ${ }^{(1,2)}, Y . \mathrm{Xu}^{(1,2)}$, J. N. Kemal ${ }^{(1)}$, A. Nesic ${ }^{(1)}, A$. Hofmann $^{(4)}$ \\ (1) Institute of Photonics and Quantum Electronics (IPQ), Karlsruhe Institute of Technology (KIT), \\ 76131 Karlsruhe, Germany \\ (2) Institute of Microstructure Technology (IMT), Karlsruhe Institute of Technology (KIT), \\ 76344 Eggenstein-Leopoldshafen, Germany \\ (3) Vanguard Photonics $\mathrm{GmbH}, 76344$ Eggenstein-Leopoldshafen, Germany \\ (4) Institute for Automation and Applied Informatics (IAI), Karlsruhe Institute of Technology (KIT), \\ 76344 Eggenstein-Leopoldshafen, Germany \\ (*) E-mail: christian.koos@kit.edu
}

Abstract We give an overview of our research towards exploiting direct-write $3 D$ laser lithography as a tool for advanced photonic integration. The technique offers new perspectives for a wide variety of applications, ranging from photonic wire bonding and multi-chip integration of high-speed communication engines to facet-attached beam-shaping elements and highly efficient coupling in astrophotonic systems. We are currently working on transferring the concept from laboratory demonstrations to industrial manufacturing.

\section{Summary}

Single-mode chip-to-chip and fiber-to-chip interfaces represent a key challenge in packaging and assembly of photonic integrated systems [1]. On the optical side, this challenge comprises two main aspects: First, on-chip waveguides frequently feature small mode-field diameters, in particular when high index-contrast silicon-photonic ( $\mathrm{SiP}$ ) or InP-based components are involved. Low-loss coupling hence requires highest positioning accuracy of optical components, which often relies on comparatively complex and expensive active alignment techniques. Second, optical interfaces have to cope with vastly different mode field profiles that need to be carefully adapted for efficient coupling.

In this presentation, we will give an overview of our research towards harnessing threedimensional (3D) nanoprinting techniques to overcome the various challenges in photonic packaging and system assembly. We have demonstrated that direct-write two-photon lithography is perfectly suited for connecting photonic chips by 3D free-form single-mode waveguides, so-called photonic wire bonds [2] [5]. This concept does not only allow to combine the distinct advantages of photonic integration platforms in high-performance hybrid multi-chip modules (MCM) [3] [4], but also greatly simplifies level-1 packaging and fiber-chip coupling of photonic integrated circuits (PIC) [5]. We demonstrated the viability of the concept by realizing multi-chip transmitter modules that combine silicon photonic modulators with InPbased laser-sources in highly integrated assemblies [6], [7]. Using the same lithography techniques, we have shown high-precision in- situ printing of lenses to facets of photonic chips and optical fibers [8] - [10]. This approach does not only allow for low-loss coupling of PIC to free-space beams in a wide variety of optical assemblies, but also opens novel opportunities in other areas such as astrophotonics, where efficient coupling between free-space beams and optical fibers is essential [10]. Taking a longterm view, on-chip 3D freeform waveguides [11] might allow large-scale non-planar circuit topologies that are, e. g., at the heart of advanced optical switches. Based on substantial research efforts carried out over the previous years, we are currently working on transferring the technology to application in industrial production processes.

\section{Acknowledgements}

This work was supported by the BMBF joint projects PRIMA (grant 13N14630) and PHOIBOS (grant 13N12574), by the H2020 Photonic Packaging Pilot Line PIXAPP (\# 731954), by the European Research Council (ERC Starting Grant 'EnTeraPIC', \# 280145; ERC Consolidator Grant 'TeraSHAPE', \# 773248), by the Deutsche Forschungsgemeinschaft in the framework of the collaborative research center "WavePhenomena" (CRC 1173), by the Alfried Krupp von Bohlen und Halbach Foundation, by the Karlsruhe NanoMicro Facility (KNMF), by the Karlsruhe School of Optics and Photonics (KSOP), and by the Helmholtz International Research School for Teratronics (HIRST).

\section{References}

[1] Lee, J. S.; Carrlo, L.; Scarcalla, C.; Pavarelli, N.; Menezo, S.; Bernabé, S.; Temporiti, E.; O'Brien, P.: 'Meeting the electrical, optical, and thermal design 
challenges of photonic-packaging;' IEEE J. Sel. Topics Quantum Electron. 22, 409-417 (2016).

[2] Lindenmann, N.; Balthasar, G.; Hillerkuss, D.; Schmogrow, R.; Jordan, M.; Leuthold, J.; Freude, W.; Koos, C.: 'Photonic wire bonding: a novel concept for chip-scale interconnects;' Opt. Express 20, 1766717677 (2012)

[3] Billah, M. R.; Blaicher, M.; Hoose, T.; Dietrich, P.-I.; Marin, P.; Lindenmann, N.; Nesic, A.; Hofmann, A.; Troppenz, U.; Moehrle, M.; Randel, S.; Freude, W.; Koos, C.: "Hybrid integration of silicon photonics circuits and InP lasers by photonic wire bonding"; arXiv:1802.03454 [physics.app-ph]

[4] Hoose, T.; Billah, M.; Marin, P.; Blaicher, M.; Dietrich, P.; Hofmann, A.; Troppenz, U.; Möhrle, M.; Lindenmann, N.; Thiel, M.; Simon, P.; Hoffmann, J.; Goedecke, M.; Freude, W.; Koos, C.: 'Connecting surface and edge emitting lasers to silicon chips,' Optical Fiber Communication Conference (OFC'16), Los Angeles (CA), USA, March 20-24, 2016. Paper M2I.7

[5] Lindenmann, N.; Dottermusch, S.; Goedecke, M.-L.; Hoose, T.; Billah, M.-R.; Onanuga, T.-P; Hofmann, A.; Freude, W.; Koos, C.: 'Connecting silicon photonic circuits to multicore fibers by photonic wire bonding,' J. Lightw. Technol. 33, 755-760 (2015)

[6] Billah, M. R.; Blaicher, M.; Kemal, J. N.; Hoose, T.; Zwickel, H.; Dietrich, P.-I.; Troppenz, U.; Moehrle, M.; Merget, F.; Hofmann, A.; Witzens, J.; Randel, S.; Freude, W.; Koos, C.: '8-channel $448 \mathrm{Gbit} / \mathrm{s}$ silicon photonic transmitter enabled by photonic wire bonding,' Optical Fiber Communication Conference
(OFC'17), Los Angeles (CA), USA, March 19-23, paper Th5D.6. (2017)

[7] Billah, M. R.; Kemal, J. N.; Marin-Palomo, P.; Blaicher, M.; Kutuvantavida, Y.; Kieninger, C.; Zwickel, H.; Dietrich, P.-I.; Wolf, S.; Hoose, T.; Xu, Y.; Troppenz, U.; Moehrle, M.; Randel, S.; Freude, W.; Koos, C.: 'Four-Channel 784 Gbit/s Transmitter Module Enabled by Photonic Wire Bonding and Silicon-Organic Hybrid Modulators'; 43rd European Conf. Opt. Commun. (ECOC'17), Gothenburg, Sweden, Sept. 17-21, paper Th.PDP.C.1 (2017) (Post-deadline paper)

[8] Schneider, S.; Lauermann, M.; Dietrich, P.-I.; Weimann, C.; Freude, W.; Koos, C.: 'Optical coherence tomography system mass-producible on a silicon chip'; Opt. Express 24, 1573-1586 (2016)

[9] Dietrich, P.-I.; Blaicher, M.; I. Reuter, I.; Billah, M.; T. Hoose, T.; A. Hofmann, A.; Caer, C.; Dangel, R.; Offrein, B.; Troppenz, U.; Moehrle, M.; Freude, W.; Koos, C.: 'In-Situ 3D Nano-Printing of Freeform Coupling Elements for Hybrid Photonic Integration'; Nature Photon. 12, 241-247 (2018)

[10] Dietrich, P.-I.; Harris, R. J.; Blaicher, M.; Corrigan, M. K.; Morris, T. M.; Freude, W.; Quirrenbach, A.; Koos, C.: 'Printed freeform lens arrays on multi-core fibers for highly efficient coupling in astrophotonic systems,' Opt. Express 25, 18288 - 18295 (2017)

[11] Nesic, A.; Blaicher, M.; Hoose, T.; Lauermann, M.; Kutuvantavida, Y.; Freude, W.; Koos, C.: 'Hybrid 2D/3D photonic integration for non-planar circuit topologies,' 42nd European Conf. Opt. Commun. (ECOC'16), Düsseldorf, Germany, Sept. 18-22, 2016. Paper W.3.F.4 